\title{
Leading-Edge Noise Prediction of General Airfoil Profiles with Spanwise-Varying Inflow Conditions
}

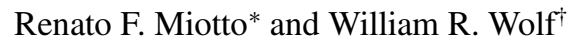 \\ University of Campinas, 13083-860 Campinas SP, Brazil \\ and \\ Leandro D. de Santana \\ University of Twente, 7522 NB Enschede, The Netherlands
}

DOI: $\underline{10.2514 / 1 . J 056716}$

\begin{abstract}
This paper presents a study of the leading-edge noise radiated by an airfoil undergoing a turbulent inflow. The noise prediction of generic airfoil profiles subjected to spanwise-varying inflow conditions is performed with the support of Amiet's theory and the inverse strip technique. In the proposed methodology, the aeroacoustic transfer function of a generic airfoil profile is computed by the boundary element method. The effects of the airfoil leading-edge thickness on the inflow turbulence are accounted for by a turbulence spectrum based on the rapid distortion theory. This research shows that the turbulence distortion plays a significant role on the predicted noise levels. Compared with the von Kármán model for isotropic turbulence, the rapid distortion theory predicts reduced noise levels at high frequencies and increased noise levels at low frequencies. This paper also shows that the spanwise-varying inflow, here represented by a linearly changing condition, contributes to raising the noise levels when compared to the similar uniform inflow case. This research confirms that the finite airfoil thickness decreases the airfoil-gust lift response, consequently reducing the noise levels. This observation is more pronounced for microphones positioned downstream of the airfoil and for high frequencies.
\end{abstract}

\section{Nomenclature}

$\mathrm{BEM}=$ boundary element method

$b \quad=\quad$ airfoil semichord, $\mathrm{m}$

$c \quad=$ airfoil chord $(2 b), \mathrm{m}$

$d \quad=$ airfoil half-span, $\mathrm{m}$

$\mathrm{FP}=$ flat plate

FIN = finite plate

$g \quad=\quad$ transfer function between the incoming gust and the airfoil pressure jump

Inf $=$ infinite span

InvS = inverse strip theory

$\mathrm{i} \quad=$ imaginary unity $(\sqrt{-1})$

$K_{2}=$ particular hydrodynamic wave number in the spanwise direction $\left(k y / r_{o}\right), \mathrm{m}^{-1}$

$k=$ acoustic wave number, $\mathrm{m}^{-1}$

$k_{e} \quad=$ average wave number of the energy-containing eddies, $\mathrm{m}^{-1}$

$k_{1}=$ hydrodynamic wave number in the chordwise direction, $\mathrm{m}^{-1}$

$k_{2}=$ hydrodynamic wave number in the spanwise direction, $\mathrm{m}^{-1}$

$\mathcal{L}=$ aeroacoustic transfer function

$M_{0} \quad=$ freestream Mach number

$\mathrm{RDT}=$ rapid distortion theory corrected model

RMS $=$ root mean square

$r_{o} \quad=$ far-field observer position, $\mathrm{m}$

$S_{p p}=$ power spectral density of the pressure fluctuation, $\mathrm{Pa}^{2} \cdot \mathrm{s}$

$t=$ time, $\mathrm{s}$

Received 12 September 2017; revision received 17 January 2018; accepted for publication 20 January 2018; published online 13 March 2018. Copyright (C) 2018 by Renato Miotto, William Wolf, and Leandro Santana. Published by the American Institute of Aeronautics and Astronautics, Inc., with permission. All requests for copying and permission to reprint should be submitted to CCC at www.copyright.com; employ the ISSN 0001-1452 (print) or 1533-385X (online) to initiate your request. See also AIAA Rights and Permissions www.aiaa.org/randp

*Graduate Student, Department of Energy. Member AIAA.

${ }^{\dagger}$ Assistant Professor, Department of Energy. Member AIAA.

${ }^{*}$ Assistant Professor, Faculty of Engineering Technology, Drienerlolaan 5. Member AIAA.

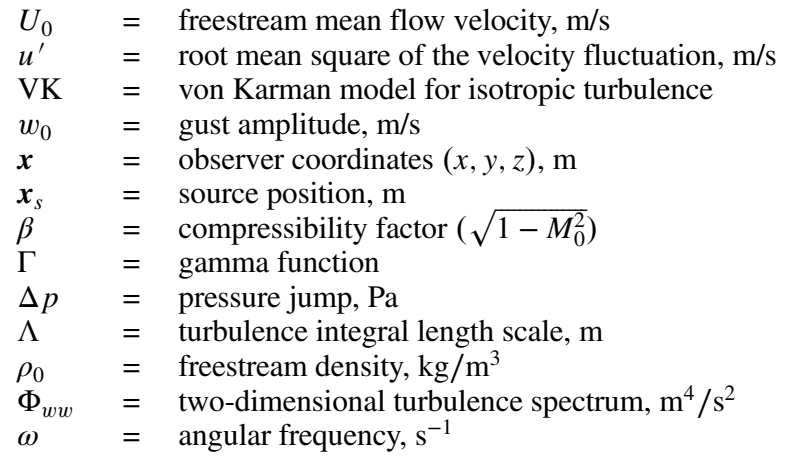

\section{Introduction}

$\mathbf{T}$ HE turbulence-airfoil interaction problem, generally regarded as an effective noise generation mechanism, is present in a wide range of technological and scientific applications [1-5]. Rotating machines such as fans, wind turbines, contrarotating open rotors, and helicopter blades are optimally designed when the noise production is already considered in the conceptual and preliminary design phase. Therefore, the development of low-computational-cost physics-based semianalytical predictive approaches that allow quick noise assessments and optimization is an instrumental tool in the industrial design process.

The determination of the unsteady pressure loading over the airfoil surface permits the application of Curle's analogy [6] for noise prediction. Following this approach, Amiet [7] assumed a flat-plate airfoil subjected to a spanwise-uniform inflow to compute the airfoil pressure distribution due to high-frequency supercritical gusts in twodimensional compressible flows. Later, numerical methodologies were proposed to account for complex incident flows and realistic airfoil geometries [으밀. Glegg and Devenport [12] adopted a panel method to predict turbulence-airfoil interaction noise, obtaining good agreement with Amiet's theory at low frequencies. Their technique, however, was not suited for high-frequency regimes where compressibility effects exert nonnegligible influence on the airfoil-gust response.

Recently, Miotto et al. [13] developed a boundary element method (BEM) for computing the aeroacoustic transfer function in the gustairfoil interaction problem. The proposed methodology allowed a study of the leading-edge noise produced due to an incident turbulent inflow interacting with general airfoil profiles, three-dimensional 
supercritical perturbations, and compressible subsonic flows. The current work proposes to combine the technique described in [13] with the inverse strip method presented by Christophe et al. [14] to predict the leading-edge noise from general airfoil profiles with spanwise-varying inflow conditions. In this research, a model based on the rapid distortion theory (RDT) $[15,16]$ is applied to account for the airfoil mean flow turbulence distortion present in the vicinity of the airfoil leading edge. Finally, the contribution of each calculation technique on the far-field noise prediction is investigated.

\section{Theoretical Background}

\section{A. Acoustic Radiation: Amiet's Theory}

The methodology proposed in this work predicts the airfoil noise, following the ideas presented by Amiet [17]. Here, we consider an airfoil of span $2 d$ and chord $2 b$ located in a Cartesian coordinate system with origin at the airfoil midchord and midspan. Following Amiet's formalism, the radiated acoustic pressure power spectral density (PSD) of a large-aspect-ratio airfoil subject to a gust represented by

$$
w=w_{0} \exp \left\{\mathrm{i}\left[k_{1}\left(U_{0} t-x\right)-k_{2} y\right]\right\}
$$

is given by

$$
S_{p p}(\boldsymbol{x}, \omega)=\left(\frac{\rho_{0} k z b}{r_{o}^{2}}\right)^{2} \pi U_{0} d \Phi_{w w}\left(k_{1}, k_{2}\right)\left|\mathcal{L}\left(\boldsymbol{x}, k_{1}, k_{2}\right)\right|^{2}
$$

where $\boldsymbol{x}=(x, y, z)$ is the observer position; $\omega$ is the frequency; $k_{1}$ and $k_{2}$ are the $x$ - and $y$-direction hydrodynamic wave numbers, respectively; and $r_{o}=\sqrt{x^{2}+\beta^{2}\left(y^{2}+z^{2}\right)}$ is the observer position corrected for convective effects, where $\beta=\sqrt{1-M_{0}^{2}}$ is the compressibility factor. The observer positions $x, y$, and $z$ are measured, respectively, in the chordwise, spanwise, and wall normal Cartesian directions. Note that $w_{0}$ is the incoming gust amplitude; $U_{0}, M_{0}$, and $\rho_{0}$ are the freestream velocity, Mach number, and density, respectively; and $k$ is the acoustic wave number.

When the airfoil thickness is considered negligible, the inflow twodimensional turbulence spectrum $\Phi_{w w}$ can be modeled by the von Kármán spectrum for isotropic turbulence [18]. On the other hand, when the airfoil thickness is no longer negligible, the rapid distortion theory can be applied $[15,16] . \mathcal{L}$ is the aeroacoustic transfer function, which can be analytically determined for a flat plate [7]. However, the aeroacoustic transfer function presents noteworthy differences for thick airfoil profiles. For example, recent research [13] shows that the airfoil thickness reduces the magnitude of the unsteady pressure distribution along the airfoil surface reducing, therefore, the magnitude of the aeroacoustic transfer function.

\section{B. Aeroacoustic Transfer Function for General Airfoil Profiles}

The aeroacoustic transfer function was defined by Amiet for an infinitely thin plate as [17]

$$
\mathcal{L}\left(\boldsymbol{x}, k_{1}, k_{2}\right)=\int_{0}^{2} g\left(x_{s}, k_{1}, k_{2}\right) \exp \left[-\mathrm{i} k b\left(x_{s}-1\right)\left(M_{0}-x / r_{o}\right) / \beta^{2}\right] \mathrm{d} x_{s}
$$

where $x_{s}$ is a particular dipole source position along the airfoil chord. Here, $g\left(x_{s}, k_{1}, k_{2}\right)$ is the transfer function that relates the airfoil unsteady pressure jump (represented by $\Delta p=p^{\text {lower }}-p^{\text {upper }}$ ) to the incident gust of amplitude $w_{0}$, and it is given by [17]

$$
g\left(x_{s}, k_{1}, k_{2}\right)=\frac{\Delta p\left(x_{s}, y_{s}, t\right) \exp \left[\mathrm{i}\left(k_{2} y-\omega t\right)\right]}{2 \pi \rho_{0} U_{0} w_{0}}
$$

The BEM approach proposed in [13] allows the calculation of the aeroacoustic transfer function accounting for general airfoil profiles. The proposed approach numerically solves the boundary value problem prescribed by the linearized airfoil theory in the transformed Prandtl-Glauert plane, providing the unsteady load distribution over the airfoil surface. This methodology considers the iterative application of the BEM calculation in an approach equivalent to consequent Schwarzschild solutions. Similar to Amiet's theory, this paper proposes the application of boundary conditions satisfying both the semi-infinite leading-edge noise problem due to an impinging gust and its subsequent semi-infinite trailing-edge scattering effect. This method is valid for blades with a large aspect ratio, subjected to three-dimensional supercritical perturbations and compressible subsonic inflow.

\section{Spanwise-Varying Inflow Conditions}

The theory initially presented by Amiet [7] considers a spanwiseuniform inflow. Hence, spanwise-varying inflow conditions bring a special challenge to the noise calculation that, here, we propose to overcome by the application of the inverse strip theory $[14,15]$. Equation (1) assumes a large-aspect-ratio airfoil with a constant spanwise section subjected to a uniform flow with homogeneous turbulence. However, in most applications of engineering interest, even if the blade section is constant, the mean flow velocity and turbulence statistics are generally nonuniform.

The most straightforward approach that considers flow spanwise inhomogeneities divides the airfoil along the spanwise direction into small strips such that the total noise corresponds to the sum of contributions from each individual strip with different flow conditions [19]. When the airfoil is discretized into small strips, the infinite-span hypothesis $k d \rightarrow \infty$ is generally not satisfied for an individual strip and Eq. (1) cannot be adopted. In this case, the more general formulation provided by Amiet [17] should be considered, where the acoustic pressure PSD requires the integration of a cardinal sine function. The reader is referred to $[14,17]$ for further details.

$$
\begin{aligned}
& S_{p p}(\boldsymbol{x}, \omega)=\left(\frac{\rho_{0} k z b}{r_{o}^{2}}\right)^{2} \pi U_{0} d \\
& \quad \times \int_{-\infty}^{+\infty} \frac{\sin ^{2}\left[d\left(k_{2}-K_{2}\right)\right]}{\pi d\left(k_{2}-K_{2}\right)^{2}} \Phi_{w w}\left(k_{1}, k_{2}\right)\left|\mathcal{L}\left(\boldsymbol{x}, k_{1}, k_{2}\right)\right|^{2} \mathrm{~d} k_{2}
\end{aligned}
$$

Christophe et al. [14] demonstrated that the direct strip approach was inaccurate in the low-frequency regime due to the short span representation inability to represent large correlations of the spanwise turbulence. If the spanwise extents of the strips were smaller than the spanwise correlation length of the sources, the strips were not statistically uncorrelated and the phase variations occurring along the span could no longer be reproduced. In this case, the increasing number of strips reduced the amplitude of the calculated far-field pressure PSD in the low-frequency portion of the spectrum and damped the oscillations appearing at high frequencies.

The inverse strip theory was then proposed in [14] to overcome the theoretical limitation of the direct strip approach. In the inverse strip method, the noise produced by an airfoil strip is calculated from the subtraction of the sound computed for two large-span airfoils, with span lengths differing by the length of the short strip segment under consideration. Therefore, this approach requires two evaluations of Eq. (1) for each strip, which is computationally inexpensive, and it produces accurate results for low and high frequencies. This method is also adequate to be applied for problems with spanwise-varying inflow conditions.

Figure 1 illustrates how the inverse strip method works. The contribution from each strip $n=1, \ldots, N$ is computed by the

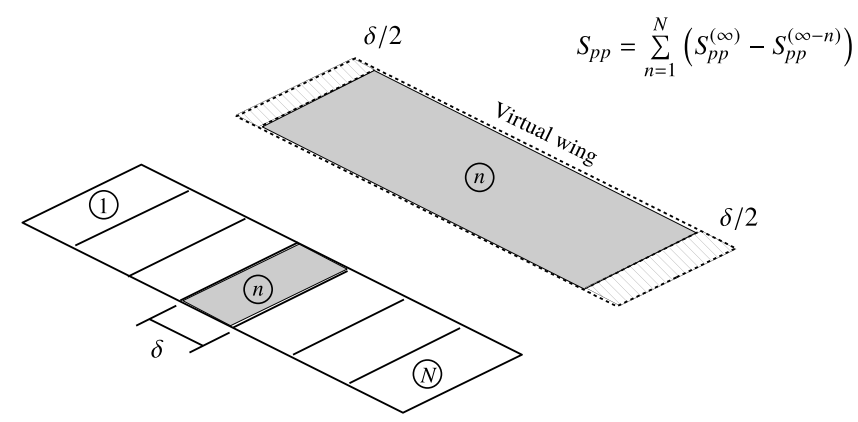

Fig. 1 Representation of the inverse strip method. 
subtraction of the pressure PSD of a large-aspect-ratio (virtual) wing using the infinite-span formulation (represented here by $S_{p p}^{(\infty)}$ ) and a second (virtual) wing at the same position, but with a span length reduced by the size $\delta$ of the strip (represented by $S_{p p}^{(\infty-n)}$ ). Both wings have the same flow properties employed on the individual strip under consideration.

\section{Rapid Distortion Theory}

When a flat plate is considered in Amiet's theory, the von Kármán spectrum for isotropic turbulence may be employed [17]:

$$
\Phi_{w w}\left(k_{1}, k_{2}\right)=\frac{4}{9 \pi} \frac{u^{\prime 2}}{k_{e}^{2}} \frac{k_{1}^{2} / k_{e}^{2}+k_{2}^{2} / k_{e}^{2}}{\left(1+k_{1}^{2} / k_{e}^{2}+k_{2}^{2} / k_{e}^{2}\right)^{7 / 3}}
$$

Here, $u^{\prime}$ stands for the rms axial velocity fluctuations and $k_{e}$ is the average wave number of the energy-containing eddies given by $k_{e}=\sqrt{\pi} \Gamma(5 / 6) /[\Lambda \Gamma(1 / 3)]$. Here, $\Gamma$ is the Gamma function and $\Lambda$ is the turbulence integral length scale.

The airfoil leading-edge bluntness induces a modification of the mean velocity field and turbulence properties [20,21]. This phenomena can be modeled by the rapid distortion theory when the timescale associated with the nonlinear turbulent interactions is much larger than the fluid particle convection through the nonuniform flow region. The turbulence intensity should also be small enough to not significantly disturb the mean flowfield. Furthermore, in the case of a turbulent flow past an airfoil leading edge, the turbulence integral correlation length scale should be larger than the boundary-layer thickness [16].

The turbulence distortion occurring close to the airfoil leading edge is related to the noise reduction observed in airfoils of increasing thickness [22]. For instance, Glegg and Devenport [23] numerically predicted the high-frequency noise reduction due to the rapid distortion of turbulence occurring in the airfoil leading edge. Likewise, a recent study from Ayton and Chaitanya [24] based on rapid distortion theory showed, by analytical and experimental means, the effect of the leading-edge radius and airfoil thickness on gust-airfoil interaction noise. For low Mach numbers, they showed that blunter leading edges led to a reduction in the far-field noise radiation. However, they also observed that, at moderate Mach numbers $\left(M_{0}=0.6\right)$, the blunter leading edge could increase farfield noise in the upstream direction for high-frequency gusts. Such a trend has been also observed in $[13,18,25]$.

Moreu et al. [26] proposed the modification of the turbulence upwash velocity spectrum by the rapid distortion theory when the airfoil thickness was no longer negligible. Following this idea, Christophe [15] derived the exact modification of the von Kármán spectrum with the rapid distortion theory. This was also summarized in the review article by Roger and Moreau [27]. In the present work, the modified von Kármán isotropic model proposed in [15] is adopted to account for the airfoil leading-edge turbulence distortion phenomena:

$$
\Phi_{w w}\left(k_{1}, k_{2}\right)=\frac{91}{36 \pi} \frac{u^{\prime 2}}{k_{e}^{2}} \frac{k_{1}^{2} / k_{e}^{2}+k_{2}^{2} / k_{e}^{2}}{\left(1+k_{1}^{2} / k_{e}^{2}+k_{2}^{2} / k_{e}^{2}\right)^{19 / 6}}
$$

Here, the important parameters obtained from experimental or numerical data are the turbulence integral length scale $\Lambda$ that should be measured away from the airfoil leading edge and the velocity fluctuations $u^{\prime}$, which should be measured in the proximity of the leading edge. Further details about this model were presented by de Santana et al. [16].

\section{Results}

The present work assumes hypothetical flow configurations to perform the noise predictions using the aforementioned techniques. In this approach, the flow quantities are chosen such that they result in a significant variation along the span direction, reproducing a scenario close to that encountered in rotating devices. The mean flow, integral length scale, and velocity fluctuation root mean square are assumed to have a linear variation along the span, such that their respective minimum and maximum values occur at the span limits. This analysis assumes the nonuniform mean flow velocity varying from 25 to $55 \mathrm{~m} / \mathrm{s}$ and the velocity fluctuation root mean square given by $u^{\prime}(y)=0.06 U(y)$, where $y \in[-d, d]$ represents the spanwise position. Finally, the integral correlation length varies linearly from 0.0125 to $0.0130 \mathrm{~m}$. These values are based on the experimental work from [16], to which the reader is referred for further details.

Acoustic predictions are computed by assuming either the previously described nonuniform inflow condition or a uniform inflow. When the uniform inflow is considered, the spanwise distribution is spatially averaged, i.e., the mean velocity is computed as

$$
U_{0}=1 /(2 d) \int_{-d}^{+d} U(y) \mathrm{d} y
$$

In the following results, we consider a uniform section wing with $c=2 b=0.1 \mathrm{~m}$ chord and $2 d=1 \mathrm{~m}$ span. Acoustic predictions are performed by considering two different far-field observer positions $\boldsymbol{x}^{(1)}=(0,0, R)$ and

$$
\boldsymbol{x}^{(2)}=\left(R \frac{\sqrt{3}}{2}, 0, R \frac{1}{2}\right)
$$

with $R=30 c$. The center of the Cartesian system is positioned in the airfoil midchord and midspan.

Figure 2a verifies the current implementation of the inverse strip method. In the present analysis, Amiet's formulation [Eq. (1)] and the inverse strip technique should provide the same noise levels when one considers a high-aspect-ratio blade modeled as a flat plate subjected to uniform inflow conditions. This figure presents the acoustic pressure PSD considering three different formulations and assuming uniform inflow conditions. Here, the inverse strip solution is compared to the results of Eq. (1) (infinite-span formulation) and Eq. (4) (finite-span formulation), where the black lines and symbols represent the results of a blade of span $2 d=1 \mathrm{~m}$. For all figures, Fin denotes finite span, Inf denotes infinite span, FP denotes flat plate, and InvS denotes inverse strip theory. In this test case, the three approaches lead to the same results in the whole frequency range, written in terms of the Helmholtz number $\mathrm{He}=k c$. To guarantee solution convergence and an industrially affordable computational cost, the number of strips is set as $N_{S}=20$ for the inverse strip method. The number of intervals of integration in Amiet's finite-span formulation [Eq. (4)] is $N_{I}=800$.

For a low-aspect-ratio wing, the sine cardinal approximation adopted in the finite- and infinite-span calculations should lead to different results [14]. This case is also illustrated in Fig. 2a, where the acoustic prediction considers a short span flat plate with $\overline{2} d=0.2 \mathrm{~m}$ (results shown in blue color). In this figure, the legend titles are analogous to those from the black lines and symbols; hence, they are omitted. The highfrequency contents of the spectra are similar for the three methodologies analyzed. However, for low frequencies, the infinite-span assumption and the inverse strip method fail to reproduce the correct solution obtained by the "finite-span" integration given by Eq. (4).

Figure $2 \mathrm{~b}$ compares the aeroacoustic transfer function solutions obtained analytically, prescribed by Amiet's theory [7] and by the BEM, both for a flat plate. Here, the flat-plate aspect ratio is set as 10 $(2 d=1 \mathrm{~m})$. The infinite-span assumption is expected to be a good approximation when the BEM calculation is applied because the finitespan formulation will be computationally expensive. Furthermore, if such an assumption is not satisfied, the evaluation of spanwise-varying inflow conditions will rely on the direct strip method through calculation of Eq. (4), which could be problematic, as was shown in [14].

Figure $2 b$ verifies the convergence of the numerical method (BEM) by comparing results with the analytic solution for a flat plate. Note that the curves labeled Inf-FP-VK in Figs. $2 \mathrm{a}$ and $2 \mathrm{~b}$ are the same. These analyses allow an assessment of the effects of nonuniform spanwise inflow quantities through the use of the inverse strip method and show that the BEM and infinite-span solutions [Eq. (1)] have an excellent agreement. The inverse strip solution, which accounts for nonuniform 


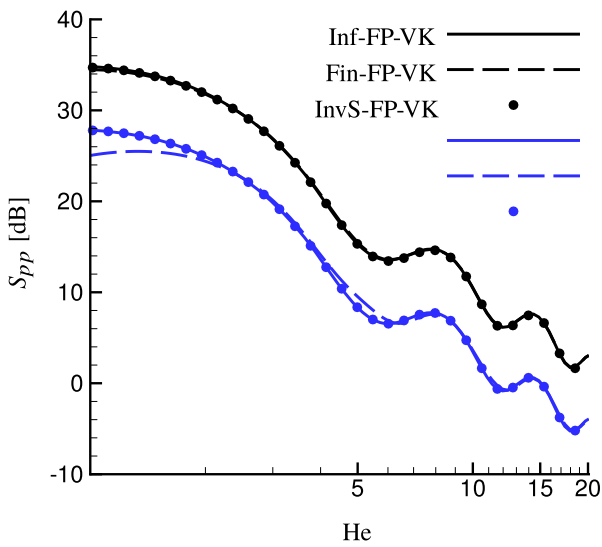

a) Spanwise-uniform inflow conditions

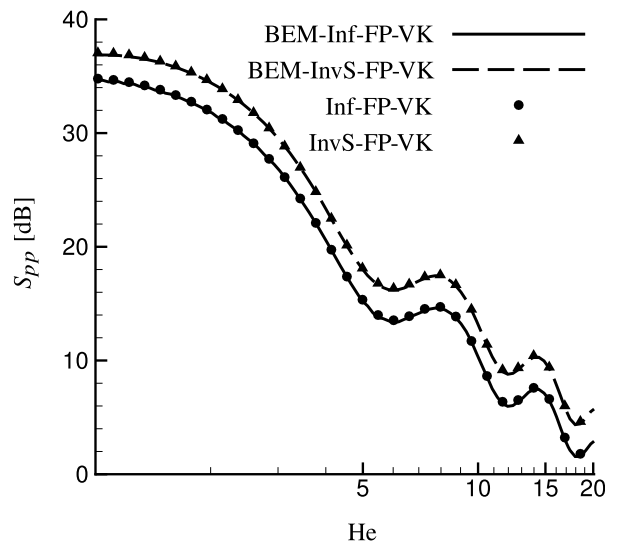

b) Spanwise-varying inflow conditions

Fig. 2 Acoustic spectra for observer $x^{(1)}$ obtained for a flat plate and the von Kármán model for isotropic turbulence. Black and blue legends indicate the long and short spans: $2 d=1.0$ and $0.2 \mathrm{~m}$, respectively.

inflows, predicts increased noise levels when compared to the infinitespan solution that considers a uniform inflow. For the current flow configuration, the predicted noise radiation increase is observed for all frequencies, reaching around $4 \mathrm{~dB}$.

The numerical calculation of the gust-airfoil lift response requires the application of boundary conditions analogous to the Schwarzschild problem [13]. As shown in the previous reference, this procedure requires a computational domain extending from beyond the airfoil limits. Here, to assure that the low frequencies are properly solved, two different domain limits are employed: $x_{s} \in[-7,9]$ (using 5000 boundary elements) for frequencies higher than $\mathrm{He}=5$, and $x_{s} \in$ $[-15,17]$ (using 10,000 boundary elements) for lower frequencies. The thickness of the extended computational domain is set to $t=0.008 b$ in the BEM calculation (see [13] for details). The numerical approach used here to compute the aeroacoustic transfer function performs two iterations of the analogous Schwarzschild problem, including the leading-edge scattering and the trailing-edge backscattering. Such an approach shows good results for moderate and high frequencies; however, the method presents discrepancies for low Helmholtz numbers $(\mathrm{He} \lesssim 1)$, especially for oblique gusts [28]. In this previous case, more iterations of the Schwarzschild problem should be conducted.

As mentioned in Secs. II.A and II.D, when nonzero thickness airfoils are considered, the flow anisotropy and the reduction in the magnitude of the aeroacoustic transfer function may significantly contribute to the far-field noise prediction. Figure 3 shows how these effects alter the acoustic pressure PSD, whether analyzed independently or combined. Figure 3 a presents results for a uniform inflow where the aeroacoustic transfer functions are obtained by the analytic solution for a flat plate, indicated by the solid lines. The aeroacoustic transfer functions are also computed for a NACA 0012 airfoil via the BEM solution, presented in dashed lines. For both cases, the von Kármán isotropic model (denoted VK) and the modified model through rapid distortion theory (denoted RDT in the figure) are assessed in the predictions. Figure $\underline{3 b}$ shows similar results, however, considering a nonuniform inflow.

For the present flow configuration, the turbulence distortion occurs close to the airfoil leading edge exhibiting the noise reduction expected by Glegg and Devenport [23] in the high-frequency range of the spectra. However, a notable increase in the noise radiation occurs at low frequencies when the RDT is accounted for. The aeroacoustic transfer function $\mathcal{L}$ obtained numerically for the NACA 0012 airfoil reduces the acoustic pressure PSD in a general sense, except for $H e \simeq 6$, where the $S_{p p}$ remains unaltered from that of the flat plate. However, this is merely due to the specific observer location. Finally, by comparing Figs. $\underline{3 \mathrm{a}}$ and $\underline{3 \mathrm{~b}}$, the uniform and spanwise-varying inflow conditions are compared, showing an increase of the PSD levels of about 2 to $4 \mathrm{~dB}$ for the latter case.

Figure 4 compares the PSDs obtained by different aeroacoustic transfer functions computed for a flat plate, a NACA 0012, a modified NACA 0012-103 [29], and a NACA 0018 airfoil. All transfer functions are computed by the proposed BEM approach. The modified spectrum for the RDT and the inverse strip method are included in this analysis to bring a complete physics-based noise prediction. For the observer location $\boldsymbol{x}^{(1)}$ (Fig. 4a), the results in terms of the flat plate and the NACA 0012 are identical to those presented in Fig. 3b. They are repeated here to allow a better assessment of the airfoil thickness effect because the NACA 0018 and the modified four-digit series NACA 0012-103 profile are also included. In this case, the numerical evaluation of $\mathcal{L}$ for realistic airfoils leads to a noise reduction in almost all the spectral range, with a maximum reduction of nearly $4 \mathrm{~dB}$. Hence, it has the same order of importance of the spanwise-varying inflow.

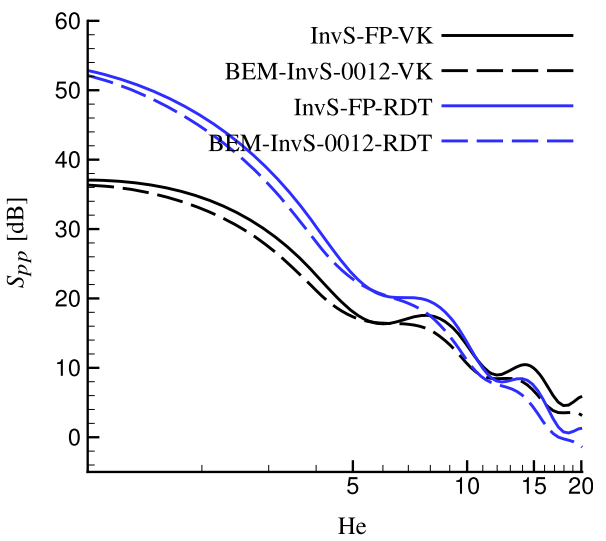

b) Inverse strip method (non-uniform inflow)

a) Infinite span formulation (uniform inflow)

Fig. 3 Spectra measured at $x^{(1)}$ for a NACA 0012 profile, comparing the influence of the von Kármán isotropic model, the modified model through rapid distortion theory, and the modified aeroacoustic transfer function from the BEM. 


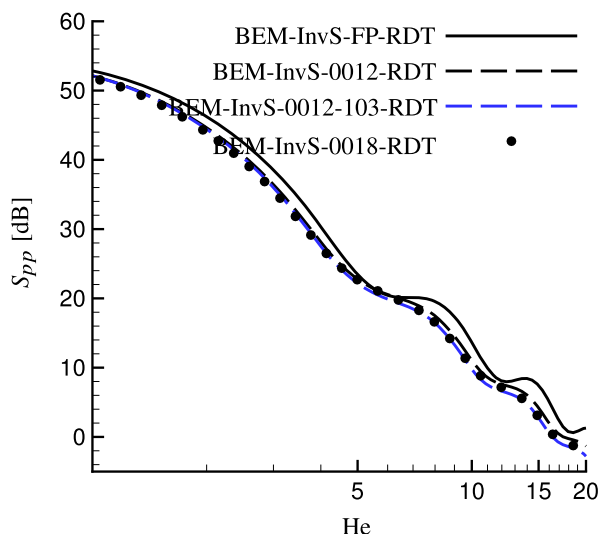

a) Observer at $\mathbf{x}^{(1)}$

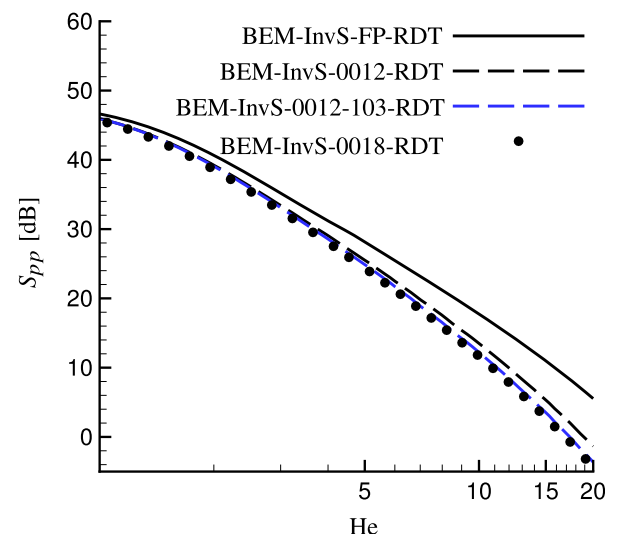

b) Observer at $\mathbf{x}^{(2)}$

Fig. 4 Comparison of spectra obtained for a flat plate, a NACA 0012, a modified NACA 0012-103, and a NACA 0018 profile using the modified von Kármán model with RDT and the inverse strip method, and considering a nonuniform inflow.

A second observer location at $\boldsymbol{x}^{(2)}$ is considered in Fig. 4b. This observer is placed at a $30 \mathrm{deg}$ angle with respect to the chordwise direction, corresponding to a region of the scattered acoustic field where more pronounced lobes appear in the pressure directivities for the higher Helmholtz numbers analyzed. In this case, the aeroacoustic transfer function has a higher impact in the noise prediction, especially at high frequencies. This trend has been observed in previous work $[13,18,24-26]$, and it is due to the fact that the directivity changes from the typical cardioid shape to that of compact dipole source for thicker airfoil profiles. In this sense, for the flat plate, the lobes are more pronounced at this observer location and, when the finite thickness effect is accounted for, there is a reduction in the noise radiation at this observer position.

In the present work, we also employ a NACA 0012-103 (which is a modified NACA four-digit series) to analyze the effect of increasing the airfoil leading-edge radius but maintaining the overall thickness of the airfoil constant. The result for this case, shown in Fig. 4 in a blue color, reviews that the modification of the aeroacoustic transfer function for such a profile reduces the noise radiation for all frequencies and observer positions analyzed when compared to the standard NACA 0012. The blunter leading edge leads to an analogous effect of increasing thickness because the spectrum is quite close to that of the NACA 0018. In this sense, the trends obtained in this work are similar to those from [24], showing that the leading-edge radius plays an interesting alternative for reducing the gust-airfoil interaction noise.

\section{Conclusions}

This work investigates the contribution from different methodologies employed together with Amiet's theory for the leading-edge noise prediction. The current methodology allows the noise prediction of general airfoil profiles with spanwise-varying inflow conditions.
The inverse strip method for nonuniform flows is combined with the rapid distortion theory to calculate the two-dimensional turbulence spectrum, and the boundary element method is employed to compute the aeroacoustic transfer function of general airfoil profiles.

It is shown that the RDT plays the most significant role on far-field noise prediction. For high frequencies, when the airfoil can be regarded as a noncompact source, the RDT model leads to a reduction in the radiated far-field noise when compared to the von Kármán model for isotropic turbulence. A similar observation is found in the literature. However, for the low-frequency range, noise levels are considerably increased with the RDT model. The spanwise-varying inflow conditions analyzed in the current work are found to raise the pressure PSD from 2 to $4 \mathrm{~dB}$ when compared to the uniform inflow case investigated. On the other hand, the airfoil-gust lift response computed for an airfoil with finite thickness is shown to reduce the noise levels, especially for observer positions downstream of the airfoil and for higher frequencies. The results also show that a blunter leading edge generates an analogous effect that is similar to that of increasing the airfoil thickness, i.e., reduction of the far-field noise.

\section{Appendix: Validation of the Methodology}

In this appendix, we combine the modifications used in this paper for Amiet's theory and apply the resulting model for a jet-airfoil interaction noise problem. The results are then compared to those presented in [15]. Here, the problem consists of a NACA 0012 profile with $c=2 b=0.041 \mathrm{~m}$ chord and $2 d=8.78 c$ span placed at a zero angle of attack and at $6 c$ distance from the jet outlet. The jet diameter and the airfoil chord both have the same length, and the jet outlet velocity $U_{0}$ is fixed at $13.2 \mathrm{~m} / \mathrm{s}$, resulting in a flow Reynolds number close to 36,000 based on the airfoil chord length. A commercial software is used to simulate the unsteady turbulent flow around the

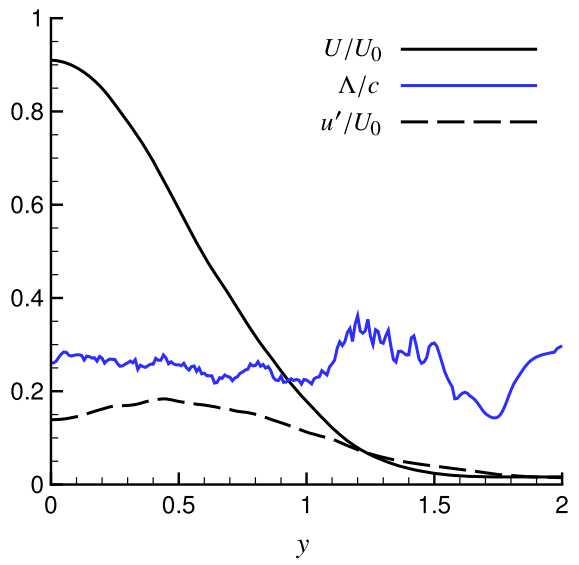

a) Autocorrelations

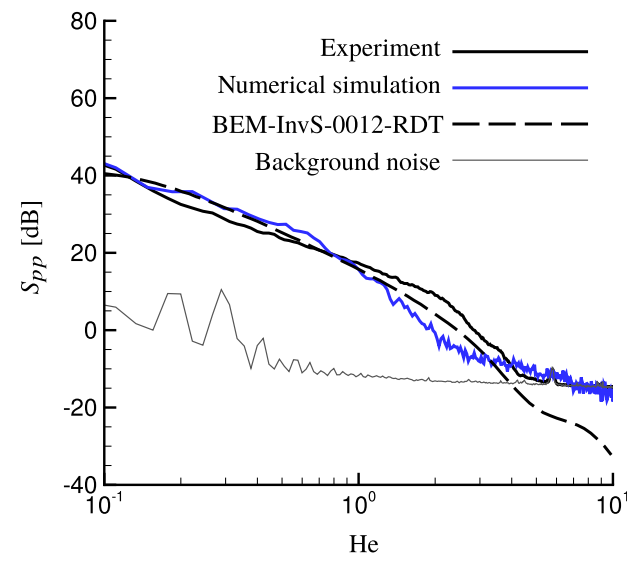

b) Noise spectra

Fig. A1 Comparison of spectra obtained for a jet-airfoil noise interaction using a NACA 0012 profile. 
airfoil, and the far-field sound spectrum of the numerical simulation is obtained through Curle's formulation. The far-field measurements are performed at an observer distance of $4.878 c$ from the airfoil center. In addition, an experiment is conducted under similar geometrical and flow conditions. The reader is referred to [15] for details about both the numerical and experimental procedures.

In Fig. A1a, the mean velocity, the integral length scale, and the turbulence intensity profiles from [15] are plotted. Figure A1b, in turn, shows the noise spectra obtained by the different approaches. The combined model used in the present work, indicated as BEMInvS-0012-RDT, shows a good agreement to the experimental and numerical results from [15], validating the present methodology. We also observe that, at the highest frequencies, the experimental noise spectrum is dominated by the background noise, causing the mismatch between the current numerical model and the other results.

\section{Acknowledgments}

The authors gratefully acknowledge the support for this research provided by Fundação de Amparo à Pesquisa do Estado de São Paulo (FAPESP) under research grants 2013/03413-4 and 2013/ 07375-0, and by Conselho Nacional de Desenvolvimento Científico e Tecnológico under research grant 305277/2015-4. The computational resources provided by Centro Nacional de Processamento de Alto Desempenho under project 551 are also gratefully acknowledged. The authors also acknowledge FAPESP for providing M.Sc. and Ph.D. scholarships to the first author under grants 2015/19538-6 and 2017/ 10795-1, respectively. Finally, the 4TU Research Centre of Fluid and Solid Mechanics is acknowledged for a one month working visit of Renato Miotto and William Wolf to the Netherlands, during which part of this work was done. The authors gratefully acknowledge Julien Christophe for providing the data used in one of the figures.

\section{References}

[1] Glegg, S., Baxter, S., and Glendinning, A., "The Prediction of Broadband Noise from Wind Turbines," Journal of Sound and Vibration, Vol. 118, No. 2, 1987, pp. 217-239. doi:10.1016/0022-460X(87)90522-0

[2] Blandeau, V. P., Joseph, P. F., Jenkins, G., and Powles, C. J., "Comparison of Sound Power Radiation from Isolated Airfoils and Cascades in a Turbulent Flow," Journal of the Acoustical Society of America, Vol. 129, No. 6, 2011, pp. 3521-3530. doi: $10.1121 / 1.3569706$

[3] Kim, J. W., Haeri, S., and Joseph, P. F., "On the Reduction of AerofoilTurbulence Interaction Noise Associated with Wavy Leading Edges," Journal of Fluid Mechanics, Vol. 792, April 2016, pp. 526-552. doi: $10.1017 / \mathrm{jfm} .2016 .95$

[4] Giez, J., Vion, L., Roger, M., Yakhina, G., and Moreau, S., "Effects of Intermittency and Geometry on the Turbulence Impingement Noise of a CROR Rear-Rotor Blade," 23rd AIAA/CEAS Aeroacoustics Conference, AIAA Paper 2017-3217, July 2017. doi:10.2514/6.2017-3217

[5] Quaglia, M., Leonard, T., Moreau, S., and Roger, M., "A 3D Analytical Model for Orthogonal Blade-Vortex Interaction Noise," Journal of Sound and Vibration, Vol. 399, 2017, pp. 104-123. doi:10.1016/j.jsv.2017.03.023

[6] Curle, N., "The Influence of Solid Boundaries Upon Aerodynamic Sound," Proceedings of the Royal Society of London, Series A: Mathematical and Physical Sciences, Vol. 231, No. 1187, 1955, pp. 505-514. doi:10.1098/rspa.1955.0191

[7] Amiet, R. K., "High Frequency Thin-Airfoil Theory for Subsonic Flow," AIAA Journal, Vol. 14, No. 8, 1976, pp. 1076-1082. doi: $10.2514 / 3.7187$

[8] Gennaretti, M., Luceri, L., and Morino, L., "A Unified Boundary Integral Methodology for Aerodynamics and Aeroacoustics of Rotors," Journal of Sound and Vibration, Vol. 200, No. 4, 1997, pp. 467-489. doi:10.1006/jsvi.1996.0713

[9] Guidati, G., and Wagner, S., "The Influence of Airfoil Shape on GustAirfoil Interaction Noise in Compressible Flows," Aeroacoustics Conferences, AIAA Paper 1999-1843, 1999. doi: $10.2514 / 6.1999-1843$

[10] Zhou, Q., and Joseph, P., “A Frequency Domain Numerical Method for Airfoil Broadband Self-Noise Prediction," Journal of Sound and Vibration, Vol. 299, No. 3, 2007, pp. 504-519. doi:10.1016/j.jsv.2006.06.061
[11] Gennaretti, M., Testa, C., and Bernardini, G., "An Unsteady Aerodynamic Formulation for Efficient Rotor Tonal Noise Prediction," Journal of Sound and Vibration, Vol. 332, No. 25, 2013, pp. 6743-6754. doi:10.1016/j.jsv.2013.07.024

[12] Glegg, S. A., and Devenport, W. J., "Panel Methods for Airfoils in Turbulent Flow," Journal of Sound and Vibration, Vol. 329, No. 18, 2010, pp. 3709-3720.

doi:10.1016/j.jsv.2010.03.007

[13] Miotto, R. F., Wolf, W. R., and Santana, L. D., "Numerical Computation of Aeroacoustic Transfer Functions for Realistic Airfoils," Journal of Sound and Vibration, Vol. 407, Oct. 2017, pp. 253-270. doi:10.1016/j.jsv.2017.06.037

[14] Christophe, J., Anthoine, J., and Moreau, S., "Amiet's Theory in Spanwise-Varying Flow Conditions," AIAA Journal, Vol. 47, No. 3, 2009, pp. 788-790. doi: $10.2514 / 1.37502$

[15] Christophe, J., "Application of Hybrid Methods to High Frequency Aeroacoustics," Ph.D. Thesis, von Kármán Inst. for Fluid Dynamics, Sint-Genesius-Rode, Belgium 2011.

[16] de Santana, L. D., Christophe, J., Schram, C., and Desmet, W., "A Rapid Distortion Theory Modified Turbulence Spectra for Semi-Analytical Airfoil Noise Prediction," Journal of Sound and Vibration, Vol. 383, Nov. 2016, pp. 349-363. doi:10.1016/j.jsv.2016.07.026

[17] Amiet, R., "Acoustic Radiation from an Airfoil in a Turbulent Stream," Journal of Sound and Vibration, Vol. 41, No. 4, 1975, pp. 407-420. doi:10.1016/S0022-460X(75)80105-2

[18] Paterson, R. W., and Amiet, R. K., "Noise and Surface Pressure Response of an Airfoil to Incident Turbulence," Journal of Aircraft, Vol. 14, No. 8, 1977, pp. 729-736. doi: $10.2514 / 3.58845$

[19] Rozenberg, Y., Roger, M., Guédel, A., and Moreau, S., "Rotating Blade Self Noise: Experimental Validation of Analytical Models," Aeroacoustics Conferences, AIAA Paper 2007-3709, 2007. doi:10.2514/6.2007-3709

[20] Batchelor, G. K., and Proudman, I., "The Effect of Rapid Distortion of a Fluid in Turbulent Motion," Quarterly Journal of Mechanics and Applied Mathematics, Vol. 7, No. 1, 1954, pp. 83-103. doi:10.1093/gjmam/7.1.83

[21] Hunt, J. C. R., "A Theory of Turbulent Flow Round Two-Dimensional Bluff Bodies," Journal of Fluid Mechanics, Vol. 61, No. 4, 1973, pp. 625-706. doi:10.1017/S0022112073000893

[22] Migliore, P., and Oerlemans, S., "Wind Tunnel Aeroacoustic Tests of Six Airfoils for Use on Small Wind Turbines," National Renewable Energy Lab. TR NREL/CP-500-35090, 2003. doi:10.1115/1.1790535

[23] Glegg, S. A., and Devenport, W., "Unsteady Loading on an Airfoil of Arbitrary Thickness," Journal of Sound and Vibration, Vol. 319, Nos. 3-5, 2009, pp. 1252-1270. doi:10.1016/j.jsv.2008.06.053

[24] Ayton, L. J., and Chaitanya, P., "Analytical and Experimental Investigation into the Effects of Leading-Edge Radius on Gust-Aerofoil Interaction Noise," Journal of Fluid Mechanics, Vol. 829, Oct. 2017, pp. 780-808. doi:10.1017/jfm.2017.594

[25] Lockard, D. P., and Morris, P. J., "Radiated Noise from Airfoils in Realistic Mean Flows," AIAA Journal, Vol. 36, No. 6, 1998, pp. 907-914. doi:10.2514/2.494

[26] Moreau, S., Roger, M., and Jurdic, V., "Effect of Angle of Attack and Airfoil Shape on Turbulence-Interaction Noise," 11th AIAA/CEAS Aeroacoustics Conference (26th AIAA Aeroacoustics Conference), AIAA Paper 2005-2973, 2005. doi: $10.2514 / 6.2005-2973$

[27] Roger, M., and Moreau, S., "Extensions and Limitations of Analytical Airfoil Broadband Noise Models," International Journal of Aeroacoustics, Vol. 9, No. 3, 2010, pp. 273-305. doi:10.1260/1475-472X.9.3.273

[28] Miotto, R. F., Wolf, W. R., and de Santana, L. D., "Numerical Computation of Airfoil-Gust Lift Response with Applications to Leading-Edge Noise Generation," 23rd AIAA/CEAS Aeroacoustics Conference, AIAA Paper 2017-3693, 2017. doi:10.2514/6.2017-3693

[29] Ladson, C. L., Brooks, C. W., Jr., Hill, A. S., and Sproles, D. W., "Computer Program to Obtain Ordinates for NACA Airfoils," NASATR 4741, Dec. 1996. 